\title{
Some Recent Trends in Motivic Homotopy Theory
}

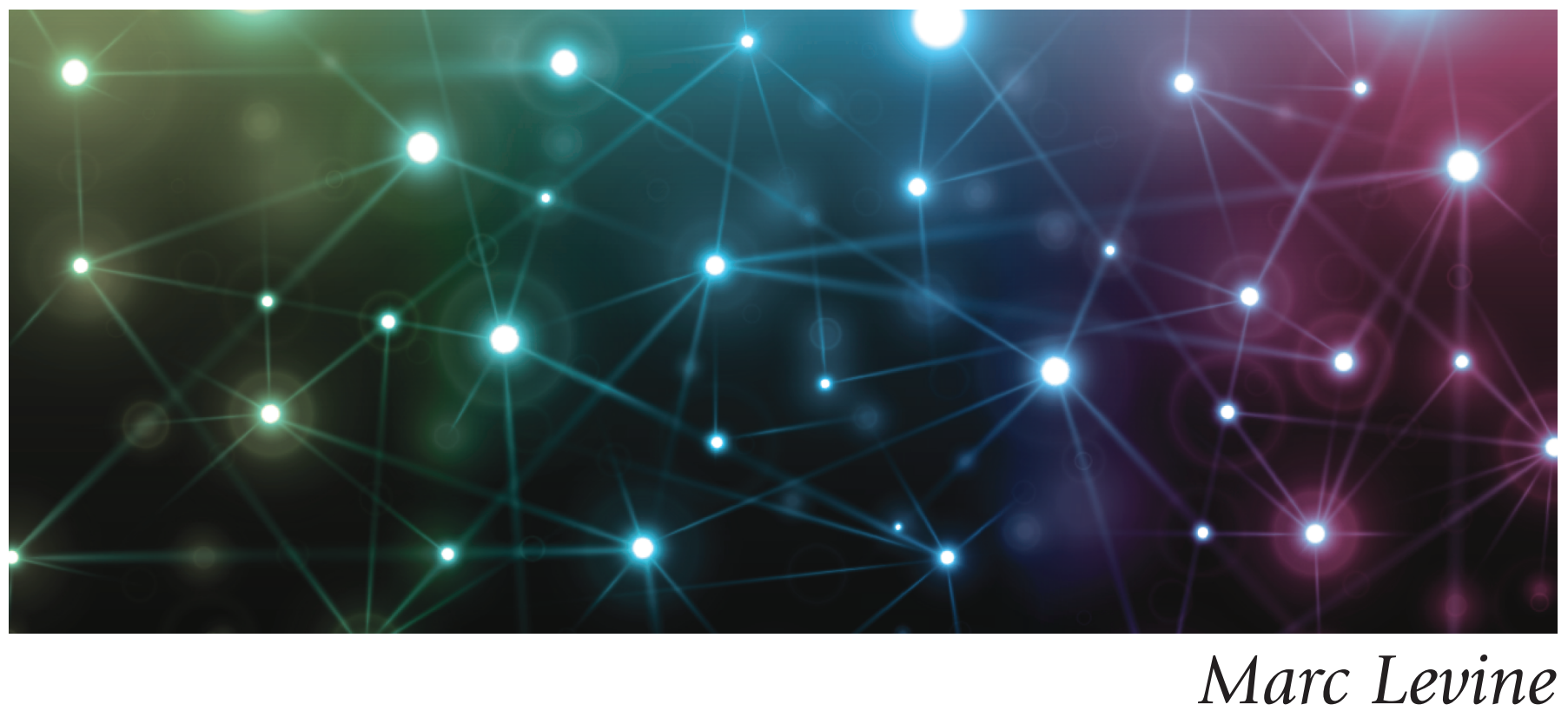

\section{Introduction}

Since its inception in the 1990s by Morel and Voevodsky, and Voevodsky's application to the proofs of the Milnor conjecture and the Bloch-Kato conjecture, $\mathbb{A}^{1}$-homotopy theory or, as it is also known, motivic homotopy theory has experienced an explosive development. I had planned in this survey to give an overview of progress and vistas in motivic homotopy theory as it has developed in the past ten years or so. I was both dismayed and heartened to find that such an undertaking in the limited space available was quite impossible: there has simply been too much going on to report on in this format. I was therefore compelled to select a few of the many areas I had planned to discuss for inclusion in this article. For this reason, I have not written anything at all about such topics as the recent applications of the various Postnikov-like towers in motivic homotopy theory; the Calmés-Fasel theory of Chow-Witt motives

Marc Levine is a professor of mathematics at Universität Duisburg-Essen. His email address is marc. 1evine@uni-due.de.

The author would like to thank the DFG for its support through the SFB Transregio 45 and the SPP 1786 "Homotopy theory and algebraic geometry."

Communicated by Notices Associate Editor Daniel Krashen.

For permission to reprint this article, please contact:

reprint-permission@ams.org.

DOI: https://doi.org/10.1090/noti2009 and its relation to the motivic sphere spectrum; the beautiful work of Ananyevskiy-Druzhinin-Garkusha-PaninNeshitov-Sosnilo and Elmanto-Hoyois-Khan-SosniloYakerson on motivic recognition principles; the study of the tensor triangulated geometry of the motivic stable homotopy category by Andrews, Gheorghe, Heller, Hornbostel, Joachimi, Kelly, and Thornton; connections with equivariant homotopy theory by $\mathrm{Hu}$, Heller, Kriz, Ormsby, and others; the computations of unstable homotopy groups with applications to splitting vector bundles by Asok and Fasel; or Ayoub's work on motives for integrable connections. Some of these topics are covered in my memorial article for Vladimir Voevodsky in the Bulletin; others may be found in the lovely survey article [7] of Isaksen-Østvær, as well as many other articles and workshop notes. Nonetheless, I hope that this perhaps idiosyncratic selection of mine will still be of interest to those nonexperts who would like to get a taste of a few of the many directions in which motivic homotopy theory is moving today. Finally, I would like to thank the AMS for giving me the opportunity to present this material to the mathematical community and the referees for their very helpful comments and suggestions. 


\section{What Is Motivic Homotopy Theory?}

In a nutshell, motivic homotopy theory is a particular homotopy theory of algebraic varieties. Motivic homotopy is distinguished from other homotopical approaches to algebraic geometry, which rely on constructions such as the homotopy theory of complex algebraic varieties or the étale homotopy theory of schemes, by being essentially internal. That is, motivic homotopy theory does not rely on giving individual algebraic varieties a topology (or Grothendieck topology) and constructing thereby a homotopy type in the usual homotopy theory of topological spaces, but builds new categories of a homotopical nature that include algebraic varieties themselves as objects and in some sense are generated by these objects. Additionally, the affine line $A^{1}$ plays a privileged role as being a version of the classical interval, so one can mix classical notions of homotopy or homotopy equivalence with notions of $\mathbb{A}^{1}$-homotopy and $\mathbb{A}^{1}$-homotopy equivalence, which in turn may be viewed as refinements of the classical notion of rational equivalence of algebraic cycles.

We begin with a brief sketch of the main players. Classical homotopy theory begins with choosing a suitable category of spaces, Spc, for example CW complexes or, for the more combinatorially minded, simplicial sets (we will stick with this latter choice here). Given a small category $\mathcal{C}$, one can enlarge $\mathbf{S p c}$ to the functor category $\operatorname{Spc}(\mathcal{C}):=\operatorname{Fun}\left(\mathcal{C}^{\mathrm{op}}, \operatorname{Spc}\right)$, that is, presheaves of spaces on $\mathcal{C}$. Viewing the category of sets as the category of discrete spaces or constant simplicial sets, the Yoneda embedding $\mathcal{C} \hookrightarrow \operatorname{Fun}\left(\mathcal{C}^{\text {op }}\right.$, Sets) gives an embedding $\mathcal{C} \hookrightarrow \operatorname{Spc}(\mathcal{C})$, with $X \in \mathcal{C}$ going to the functor $Y \mapsto \operatorname{Hom}_{\mathcal{C}}(Y, X)=: X(Y)$. We also have the embedding $\operatorname{Spc} \hookrightarrow \operatorname{Spc}(\mathcal{C})$ sending a space $K$ to the constant presheaf on $\mathcal{C}$ with value $K$. Thus $\operatorname{Spc}(\mathcal{C})$ gives a framework for combining $\mathcal{C}$ with $\mathbf{S p c}$.

As a functor category, $\mathbf{S p c}(\mathcal{C})$ inherits many good properties of Spc, for example, products and coproducts, limits and colimits, all computed sectionwise. As Spc is generated by the set of $n$-simplices $\left\{\Delta^{n} \mid n=0,1, \ldots\right\}$, this gives us the set of generators $\left\{X \times \Delta^{n} \mid X \in \mathcal{C}, n=0,1, \ldots\right\}$ for $\operatorname{Spc}(\mathcal{C})$. One has a similar construction of pointed spaces on $\mathcal{C}, \mathbf{S p c} .(\mathcal{C})$, by taking presheaves of pointed spaces.

For a presheaf $x \in \operatorname{Spc}(\mathcal{C})$, one has the associated presheaf of sets $\pi_{0}(X), \pi_{0}(X)(x)=\pi_{0}(X(x))$, and for a pointed presheaf $x \in \mathbf{S p c}$. (C) the presheaves of groups $\pi_{n}(X)$ (for $n \geq 1$, abelian for $n \geq 2$ ). This gives the notion of a weak homotopy equivalence of pointed presheaves, namely, a map $f: x \rightarrow y$ that induces an isomorphism on $\pi_{n}$ for all $n$ (in the unpointed case, one needs to make sense of the notion of "choice of basepoint," but this can be done). This gives us the unstable homotopy category $\mathcal{H}^{p s}(\mathcal{C}):=\mathbf{S p c}(\mathcal{C})\left[W E^{-1}\right]$ and the pointed version $\mathcal{H}_{\bullet}^{p s}(\mathcal{C}):=\operatorname{Spc} .(\mathcal{C})\left[W E^{-1}\right]$ by formally inverting the respective weak equivalences. For $\mathcal{C}$ the one-point category, we have $\mathbf{S p c}(\mathcal{C})=\mathbf{S p c}, \mathcal{H}^{p s}(\mathcal{C})$ is the classical unstable homotopy category $\mathcal{H}$, and similarly for the pointed versions.

There are technical problems with this that we mention briefly: as the categories $\mathbf{S p c}(\mathcal{C})$ and $\mathbf{S p c} .(\mathcal{C})$ are not small (they contain after all Sets and Sets.), it is not clear that the localizations $\mathbf{S p c}(\mathcal{C})\left[W E^{-1}\right]$ and $\mathbf{S p c}$. $(\mathcal{C})\left[W E^{-1}\right]$ exist. Also, we would really like to have a "homotopy theory" rather than just a homotopy category. Without saying exactly what we mean by this, we want to be able to generalize the standard constructions of classical homotopy theory, such as homotopy fibers and cofibers, in this presheaf setting. There are a number of solutions to these problems; the first really successful one is based on Quillen's theory of model categories. We already have the notion of a weak equivalence in $\operatorname{Spc}(\mathcal{C})$; to have a model category structure one adds that of a fibration and cofibration, all satisfying suitable axioms. One has an even nicer structure, that of a simplicial model category, which enriches the category $\operatorname{Spc}(\mathcal{C})$ in spaces by defining the mapping space as the simplicial set

$$
\operatorname{Maps}_{\operatorname{Spc}(\mathcal{C})}(X, y)=\left[n \mapsto \operatorname{Hom}_{\mathrm{Spc}(\mathcal{C})}\left(X \times \Delta^{n}, y\right)\right] .
$$

In any case, the model category structure solves the existence problem for the homotopy categories and gives the added structures needed to define a reasonable homotopy theory of presheaves of spaces.

The motivic story starts by applying this construction in the case $\mathcal{C}=\operatorname{Sm} / S$, where $S$ is a chosen base-scheme and $\mathrm{Sm} / S$ is the category of smooth finite type $S$-schemes. This gives us the category of spaces over $S, \operatorname{Spc}(S):=\operatorname{Spc}(\mathbf{S m} / S)$, and the similarly defined category of pointed spaces over $S$, Spc. $(S)$, together with their homotopy categories $\mathcal{H}^{p s}(S)$, $\mathcal{H}^{p s}(S)$.

We have decorated the homotopy categories because these are not the categories we are ultimately looking for. In order to have some reasonable descent properties, we need to incorporate a suitable Grothendieck topology $\tau$ and we also need to make the affine line an interval-object. This is accomplished by enlarging the weak equivalences to include maps that induce isomorphisms on the associated homotopy sheaves (rather than the presheaves) and the projection maps $X \times \mathbb{A}^{1} \rightarrow X$. Again invoking the techniques of model categories, this leads to a good notion of $A^{1}-\tau$ weak equivalence $W E_{\mathbb{A}^{1}, \tau}$, homotopy categories $\mathcal{H}^{\mathbb{A}^{1}, \tau}(S):=\operatorname{Spc}(S)\left[W E_{\mathbb{A}^{1}, \tau}^{-1}\right], \mathcal{H}^{\mathbb{A}^{1}, \tau}(S):=$ Spc. $(S)\left[W E_{A^{1}, \tau}^{-1}\right]$, and associated homotopy theories. This was carried out in the seminal work of Morel-Voevodsky [17]. Recent innovations give corresponding constructions on the level of infinity categories.

The standard choice for the Grothendieck topology $\tau$ is the Nisnevich topology, where a cover of a scheme $X$ is an étale cover $\mathcal{U} \rightarrow X$ that is surjective on $L$-valued points 
for all fields $L$. We write $\mathcal{H}(S)=\mathcal{H}^{\mathbb{A}^{1}, N i s}(S), \mathcal{H} .(S)=$ $\mathcal{H}^{\mathbb{A}^{1}}, N i s(S)$. The étale topology is also useful, but we will mainly discuss the Nisnevich version.

In classical topology, one is also interested in stable phenomena, that is, information that is preserved by suspension with respect to the circle $S^{1}$. This stability property is built into the very definition of generalized (co)homology via the suspension axiom. One can also see suspension arising via Spanier-Whitehead duality, in that the SpanierWhitehead dual involves a negative suspension, so only defines a true duality after inverting the suspension operator with respect to $S^{1}$. In fact, the Spanier-Whitehead dual of a smooth compact manifold $M$ is the Thom space of its virtual normal bundle $M^{\vee}=\operatorname{Th}\left(-T_{M}\right)$, with $T_{M}$ the tangent bundle. To make some sense of this, one can find a vector bundle $\nu_{M} \rightarrow M$ and an isomorphism $\nu_{M} \oplus T_{M} \cong M \times \mathbb{R}^{n}$ for some large $n$. The Thom space of a vector bundle $V \rightarrow M$ can be defined as the disk bundle modulo the sphere bundle, so $\operatorname{Th}\left(\mathbb{R}^{n}\right)=S^{n}$. The isomorphism $\nu_{M} \oplus T_{M} \cong M \times \mathbb{R}^{n}$ translates into the "identity"

$\operatorname{Th}\left(\nu_{M}\right)=\operatorname{Th}\left(\mathbb{R}^{n}\right) \wedge \operatorname{Th}\left(-T_{M}\right)=S^{n} \wedge \mathrm{Th}\left(-T_{M}\right)=\Sigma_{S^{1}}^{n} \operatorname{Th}\left(-T_{M}\right)$.

In other words, if we had an inverse to the suspension operator $\Sigma_{S^{1}}$, we could define $\mathrm{Th}\left(-T_{M}\right):=\Sigma_{S^{1}}^{-n} \mathrm{Th}\left(\nu_{M}\right)$.

One inverts suspension by passing to a category of $S^{1}$-suspension spectra, where an object is a sequence $\left(E_{0}, E_{1}, \ldots\right)$ of pointed spaces together with bonding maps $\epsilon_{n}: E_{n} \wedge S^{1} \rightarrow E_{n+1}$, and then introducing a suitable notion of stable weak equivalence (see below). The $S^{1}$ suspension is then realized as shifting the sequence to the left, which has, as a stable inverse, the shift to the right (this replaces $E_{0}$ with the one-point space, but this new spectrum is stably equivalent to our original one).

In the motivic setting one has an analog of SpanierWhitehead duality, where instead of inverting suspension with respect to $S^{1}$, one needs to invert suspension with respect to $\mathbb{P}^{1}$. To explain how this $\mathbb{P}^{1}$ suspension arises, there is a purely algebraic definition of the Thom space of an algebraic vector bundle $V \rightarrow X$ by setting $\operatorname{Th}(V):=$ $\mathbb{P}\left(V \oplus O_{X}\right) / \mathbb{P}(V)$, where $\mathbb{P}(V) \rightarrow X$ is the bundle of projective spaces associated to the vector bundle $V \rightarrow X$. Classical excision shows that one can also use this formula to define the Thom space in the topological setting. Morally speaking, one has the same problem in the motivic setting as in the classical one: one needs to invert the operator of smash product with $\operatorname{Th}\left(\mathbb{A}_{B}^{n}\right)=\mathbb{P}_{B}^{n} / \mathbb{P}_{B}^{n-1}$ to be able to define the dual of a smooth projective $B$-scheme $X$ as $\operatorname{Th}\left(-T_{X / B}\right)$. For $n=1$, instead of $\operatorname{Th}\left(\mathbb{R}^{1}\right)=S^{1}$ we get $\operatorname{Th}\left(\mathbb{A}_{B}^{1}\right)=\mathbb{P}_{B}^{1}$ (pointed by $\infty$ ), and one can rather easily show that $\operatorname{Th}\left(\mathbb{A}_{B}^{n}\right) \cong\left(\mathbb{P}_{B}^{1}\right)^{\wedge n}$ in $\mathcal{H}$. $(B)$.

This leads to the category of $\mathbb{P}^{1}$-spectra, where a $\mathbb{P}^{1}$. spectrum is a sequence $\mathcal{E}=\left(E_{0}, E_{1}, \ldots\right)$ with $E_{n} \in \mathbf{S p c} .(S)$, together with bonding maps $\epsilon_{n}: E_{n} \wedge \mathbb{P}^{1} \rightarrow E_{n+1}$, where we consider $\mathbb{P}^{1}$ as pointed by $\infty$. This gives us the category of $\mathbb{P}^{1}$-spectra over our base-scheme $S, \operatorname{Sp}_{\mathbb{P l}}(S)$.

To define a suitable notion of stable equivalence in the topological setting, one uses the suspension map $-\wedge S^{1}: \pi_{n}(X)=\left[S^{n}, X\right]_{\mathcal{H}} . \mapsto\left[S^{n+1}, X \wedge S^{1}\right]_{\mathcal{H}} .=$ $\pi_{n+1}\left(X \wedge S^{1}\right)$, giving the stable homotopy groups $\pi_{n}(E)$ for $E=\left(\left(E_{0}, E_{1}, \ldots\right), \epsilon_{*}\right)$ a spectrum:

$$
\pi_{n}(E):=\operatorname{colim}_{N} \pi_{n+N}\left(E_{N}\right), \quad n \in \mathbb{Z},
$$

using the suspension map and bonding map $\epsilon_{N}: E_{N} \wedge$ $S^{1} \rightarrow E_{N+1}$ to define the colimit. A map $f: E \rightarrow F$ of suspension spectra is a stable weak equivalence if $f$ induces an isomorphism on $\pi_{n}$ for all $n$ and the stable homotopy category is formed from the category of suspension spectra by inverting the stable weak equivalences.

In the motivic setting, there is no nice relation of $\pi_{*}(X)$ and $\pi_{*}\left(X \wedge \mathbb{P}^{1}\right)$, but one does have the Tate circle $\mathbb{G}_{m}:=$ $\left(\mathbb{A}^{1} \backslash\{0\},\{1\}\right) \in \operatorname{Spc}_{\text {. }}(B)$ and the isomorphism in $\mathcal{H}$.(B) $\mathbb{P}^{1} \cong S^{1} \wedge \mathbb{G}_{m}$. This suggests introducing the bi-graded $\mathbb{A}^{1}$-homotopy sheaf $\pi_{a, b}^{\AA^{1}}(X)$ associated to the presheaf

$$
U \in \mathbf{S m} / B \mapsto\left[U \wedge S^{a-b} \wedge \mathbb{G}_{m}^{\wedge b}, X\right]_{\mathcal{H} .(S)}
$$

for $a \geq b \geq 0$. $\mathbb{P}^{1}$-suspension induces the map

$$
(-) \wedge \mathbb{P}^{1}: \pi_{a, b}^{\AA^{1}}(X) \rightarrow \pi_{a+2, b+1}^{\mathrm{A}^{1}}\left(X \wedge \mathbb{P}^{1}\right),
$$

and thus for a $\mathbb{P}^{1}$-spectrum $\mathcal{E}=\left(\left(E_{0}, E_{1}, \ldots\right), \epsilon_{*}\right)$ we can use $\epsilon_{N}: E_{N} \rightarrow E_{N+1} \wedge \mathbb{P}^{1}$ to define

$$
\pi_{a, b}^{\mathrm{A}^{1}}(E):=\operatorname{colim}_{N} \pi_{a+2 N, b+N}^{\mathrm{A}^{1}}\left(E_{N}\right)
$$

for all $a, b \in \mathbb{Z}$. A map $f: \mathcal{E} \rightarrow \mathcal{F}$ of $\mathbb{P}^{1}$-spectra is then defined to be a motivic stable weak equivalence if $f$ induces an isomorphism on $\pi_{a, b}^{\AA^{1}}$ for all $a, b \in \mathbb{Z}$. Inverting the motivic stable weak equivalence, one forms the motivic stable homotopy category $\mathrm{SH}(S):=\operatorname{Sp}_{\mathbb{P} 1}(S)\left[W E^{-1}\right]$. As for spaces, this localization should be put in the setting of stable model categories or stable infinity categories to have a well-defined homotopy category and a stable homotopy theory.

\section{Some Useful Motivic Cohomology Theories}

The classical stable homotopy category $\mathrm{SH}$ is the category of generalized cohomology theories, where a spectrum $E$ gives rise to the cohomology theory (on spaces $X$ ) $X \mapsto$ $E^{*}(X)$, with $E^{n}(X):=\left[\Sigma^{\infty} X_{+}, \Sigma^{n} E\right]_{\mathrm{SH}}$. Here $\Sigma^{\infty} X_{+}$is the infinite suspension spectrum $\left(X_{+}, \Sigma X_{+}, \ldots, \Sigma^{n} X_{+}, \ldots\right)$ with identity bonding maps $\epsilon_{n}$. The invertibility of $\Sigma_{\mathbb{p} 1}$ on $\mathrm{SH}(S)$ gives the two-parameter family of suspensions $\Sigma^{a, b}$ corresponding to $\Sigma_{S^{1}}^{a-b} \Sigma_{\mathbb{G}_{m}}^{b}$ for $a \geq b \geq 0$. A $\mathbb{P}^{1}$-spectrum $\mathcal{E}$ thus gives rise to bi-graded cohomology on $\mathrm{Sm} / S$ or even on Spc. $(S)$ by

$$
\begin{aligned}
& \mathcal{E}^{a, b}(X)=\left[\Sigma_{\mathbb{P} 1}^{\infty} X_{+}, \Sigma^{a, b} \mathcal{E}\right]_{\mathrm{SH}(S)}, \\
& \mathcal{E}^{a, b}(X)=\left[\Sigma_{\mathbb{P} 1}^{\infty} X, \Sigma^{a, b} \mathcal{E}\right]_{\mathrm{SH}(S)} .
\end{aligned}
$$


Here we introduce a few of the most important examples of motivic cohomology theories with their classical counterparts.

One of the first results in motivic homotopy theory was the representability of algebraic $K$-theory. In topology, stable, virtual vector bundles are represented by $\mathbb{Z} \times$ $B U, B U$ being the classifying space of the infinite unitary group. Bott periodicity extends this to a 2-periodic spectrum $K U:=(\mathbb{Z} \times B U, \Sigma \mathbb{Z} \times B U, \mathbb{Z} \times B U, \ldots)$ representing topological $K$-theory.

The unstable representability of algebraic $K$-theory was proven by Morel-Voevodsky (over a regular base-scheme) [17, Theorem 4.3.13]. One can take as model for the classifying scheme BGL / $S$ the doubly infinite Grassmannian

$$
\mathrm{Gr}_{S}=\operatorname{colim}_{n, m} \mathrm{Gr}_{S}(n, n+m) \text {. }
$$

Morel-Voevodsky first show that $\mathbb{Z} \times \mathrm{BGL} / S$ represents the Grothendieck group of algebraic vector bundles $X \mapsto K_{0}(X)$ on $\mathrm{Sm} / S$ via an isomorphism

$$
[X, \mathbb{Z} \times \mathrm{BGL} / S]_{\mathcal{H}(S)} \cong K_{0}(X)
$$

that extends to higher $K$-theory using the isomorphism

$$
K_{n}(X) \cong K_{0}\left(X \times \Delta_{\mathbb{Z}}^{n} ; X \times \partial \Delta_{\mathbb{Z}}^{n}\right)
$$

for $X$ regular and affine. Here $\Delta_{\mathbb{Z}}^{n}$ is the algebraic version of the $n$-simplex

$$
\Delta_{\mathbb{Z}}^{n}=\operatorname{Spec} \mathbb{Z}\left[t_{0}, \ldots, t_{n}\right] /\left(\sum_{i=0}^{n} t_{i}-1\right),
$$

and $\partial \Delta_{\mathbb{Z}}^{n}$ is the union of faces $t_{i}=0, i=0, \ldots, n$. Using the contractibility of $\mathbb{A}^{1}$ in $\mathcal{H}(S)$, they show that

$$
\left[S^{n} \wedge X, \mathrm{BGL} / S\right]_{\mathcal{H} .(S)} \cong K_{0}\left(X \times \Delta_{\mathbb{Z}}^{n} ; X \times \partial \Delta_{\mathbb{Z}}^{n}\right) \cong K_{n}(X),
$$

establishing the representability of algebraic $K$-theory by $\mathbb{Z} \times$ BGL $/ S$, in case of regular $S$.

This extends to a stable representability. Replacing Bott periodicity is an algebraic version: let $V_{n} \rightarrow \mathrm{BGL}_{n}=$ $\operatorname{Gr}(n, \infty)$ be the universal $n$-plane bundle and let $O(-1) \rightarrow$ $\mathbb{P}^{1}=\operatorname{Gr}(1,2)$ be the tautological line bundle. This gives the tensor product bundle $p_{1}^{*} V_{n} \otimes p_{2}^{*} O(-1) \rightarrow B \mathrm{GL}_{n} \times \mathbb{P}^{1}$. Applying the unstable representability to the virtual bundle $\left[p_{1}^{*} V_{n} \otimes p_{2}^{*} O(-1)\right]-\left[p_{1}^{*} V_{n}\right]-\left[p_{2}^{*} O(-1)\right]+\left[O_{\mathrm{BGL}_{n} \times \mathbb{P}^{1}}\right]$ gives maps $\gamma_{n}: \mathrm{BGL}_{n} / S \wedge \mathbb{P}^{1} \rightarrow \mathrm{BGL} / S$, compatible in $n$, which induce $\gamma: \mathrm{BGL} / S \wedge \mathbb{P}^{1} \rightarrow \mathrm{BGL} / S$, defining the $\mathbb{P}^{1}$ spectrum $\mathrm{KGL}:=(\mathbb{Z} \times \mathrm{BGL}, \mathbb{Z} \times \mathrm{BGL}, \ldots)$. This is explained in Voevodsky's ICM talk [18].

Another important cohomology theory represented in $\mathrm{SH}(S)$ is motivic cohomology, here for $S$ smooth over a field $k$. In the case of $S=\operatorname{Spec} k$, char $k=0$, the motivic cohomology spectrum $M \mathbb{Z}$ is represented by infinite symmetric powers, relying on a motivic analog of the Dold-Thom theorem, which for $T$ a connected pointed CW complex gives the identity $\pi_{n}\left(\operatorname{Sym}^{\infty} T\right)=\tilde{H}_{n}(T, \mathbb{Z})$.
The Dold-Thom theorem implies that the classical Eilenberg-MacLane spectrum $H \mathbb{Z}$ can be constructed as $H \mathbb{Z}=\left(\operatorname{Sym}^{\infty} S^{0}, \operatorname{Sym}^{\infty} S^{1}, \ldots, \operatorname{Sym}^{\infty} S^{n}, \ldots\right) ; H \mathbb{Z}$ represents singular cohomology on Spc: $H \mathbb{Z}^{n}(T)=H^{n}(T, \mathbb{Z})$.

Replacing $S^{1}$ with $\mathbb{P}^{1}$, one has the motivic version

$$
M \mathbb{Z}:=\left(\operatorname{Sym}^{\infty} S_{+}, \operatorname{Sym}^{\infty} \mathbb{P}^{1}, \ldots, \operatorname{Sym}^{\infty}\left(\mathbb{P}^{1}\right)^{\wedge n}, \ldots\right) .
$$

For $S=\operatorname{Spec} k, k$ a perfect field, and $X \in \mathrm{Sm} / k$, one has Voevodsky's motivic cohomology $H^{a}(X, \mathbb{Z}(b))$ defined using his triangulated category of motives $\operatorname{DM}(k)$. In case chark $=0$, this theory is represented by $M \mathbb{Z}: H^{a}(X, \mathbb{Z}(b))=$ $M \mathbb{Z}^{a, b}(X)$ (see [18]). In characteristic $p$, this still holds after inverting $p$ or by modifying the definition of $M \mathbb{Z}$ suitably. There are extensions of DM(-) to more general bases, and the results for $k$ extend to $S$ smooth over a perfect field (see for example [4]).

Both of these theories represent constructions that were available before the introduction of the motivic stable homotopy category: Quillen's higher algebraic $K$-theory goes back to the early 1970s, and motivic cohomology is represented by Bloch's higher Chow groups, first defined in the mid-1980s, while the idea that a good theory of motivic (co)homology could be achieved by a suitable algebraic translation of the Dold-Thom theorem goes back to Suslin and his construction of Suslin homology, introduced shortly after Bloch constructed his higher Chow groups. Voevodsky [18] introduced a completely new theory, algebraic cobordism, by defining the $\mathbb{P}^{1}$ spectrum MGL modeled closely on the Thom spectrum MU = $\left(\mathrm{MU}_{0}, \mathrm{MU}_{1}, \ldots\right)$. Recall that $\mathrm{MU}_{2 n}$ is the Thom space $\mathrm{Th}\left(V_{n}\right)$, where $V_{n} \rightarrow B U(n)$ is the universal rank $n$ complex vector bundle over the classifying space of the unitary group $B U(n)$. To define MGL, one simply replaces the classifying space $B U(n)$ and its universal bundle $V_{n} \rightarrow B U(n)$ with the algebraic version $E_{n} \rightarrow \mathrm{BGL}_{n}$ and the Thom space $\mathrm{MU}_{n}:=$ $\operatorname{Th}\left(V_{n}\right)$ with the algebraic Thom space $\mathrm{MGL}_{n}:=\operatorname{Th}\left(E_{n}\right)$ Completely analogous to the topological setting, the fact that the $\mathrm{MGL}_{n}$ fit together to form a $\mathbb{P}^{1}$ spectrum follows from the identity $\operatorname{Th}\left(E \oplus O_{X}\right) \cong \operatorname{Th}(E) \wedge \mathbb{P}^{1}$ for $E \rightarrow X$ a vector bundle and the fact that the restriction of $E_{n+1}$ to $\mathrm{BGL}_{n}$ is $E_{n} \oplus \mathrm{O}_{\mathrm{BGL}_{n}}$.

This opened the door to constructions of a whole slew of new cohomology theories for algebraic varieties, many of which are modeled on classical topological theories, others being strikingly new. One now has available motivic versions of all the Morava $K$-theories, cobordism theories MSL and MSp, modeled on the special linear groups and the symplectic groups, and Eilenberg-MacLane-type theories $\operatorname{EM}\left(M_{*}\right)$ built out of Morel's homotopy modules (see $[11, \$ 5.2]$ ). There are also many (in fact infinitely many) different ways of constructing families of connective covers of a given theory. We will discuss some of these in more 
detail in "Cohomology Theories, Orientations, and Characteristic Classes."

The theories $M \mathbb{Z}$, KGL, and MGL are oriented theories, which means they admit Thom isomorphisms

$$
\theta_{V}: \mathcal{E}^{a, b}(X) \stackrel{\sim}{\rightarrow} \mathcal{E}^{a+2 r, b+r}(\operatorname{Th}(V))
$$

for each rank $r$ vector bundle $V \rightarrow X$. This motivic notion is an algebraic analog of the topological theory of complex oriented spectra. In the topological setting, giving a spectrum $E$ a complex orientation gives rise to pushforward maps in E-cohomology for proper maps endowed with a complex structure on the stable normal bundle, for instance, for any proper complex analytic map of complex manifolds. In the algebraic setting, an oriented spectrum $\mathcal{E}$ gives rise to pushforward maps in $\mathcal{E}$-cohomology for proper maps of smooth schemes.

Of course, a rank $r$ vector bundle is just a Zariski locally trivial fiber bundle with fiber $\mathbb{A}^{r}$ and group $\mathrm{GL}_{r}$. One can look at "structured" vector bundles, where the extra structure is given by restricting the structure group. For instance, a rank $r$ SL-vector bundle is a vector bundle with group $\mathrm{SL}_{r}$, a rank $2 r$ symplectic bundle is a vector bundle with group $\mathrm{Sp}_{2 r} \subset \mathrm{GL}_{r}$, and so on. One can describe these structures more intrinsically: an SL-vector bundle is a vector bundle $V \rightarrow X$ together with an isomorphism $\operatorname{det} V \stackrel{\sim}{\rightarrow} O_{X}$, and a symplectic vector bundle is a vector bundle together with a symplectic form, i.e., a section $\omega$ of $\Lambda^{2} V^{\vee}$ that is nondegenerate on each fiber.

Each of these structures gives rise to a notion of orientation. An SL-oriented theory is a commutative ring spectrum $\mathcal{E} \in \mathrm{SH}(k)$ together with Thom isomorphisms

$$
\theta_{V, \rho}: \mathcal{E}^{*, *}(X) \rightarrow \mathcal{E}^{2 r+*, r+*}(\operatorname{Th}(V))
$$

for each rank $r$ vector bundle $V \rightarrow X$ with trivialized determinant bundle $\rho: \operatorname{det} V \stackrel{\sim}{\rightarrow} O_{X}$. These must satisfy a few natural axioms. An Sp-oriented theory $\mathcal{E} \in \mathrm{SH}(k)$ together with Thom isomorphisms

$$
\theta_{V, \omega}: \mathcal{E}^{*, *}(X) \rightarrow \mathcal{E}^{2 r+*, r+*}(\operatorname{Th}(V))
$$

for each rank $r$ symplectic vector bundle $(V \rightarrow X, \omega \in$ $\left.H^{0}\left(X, \Lambda^{2} V^{\vee}\right)\right)$. The corresponding cobordism theories are MSL and MSp. For the sake of uniformity of notation, we sometimes refer to an oriented theory as a GL-oriented theory.

An important example of an SL-oriented theory is hermitian K-theory. This may be constructed as a version of Quillen $K$-theory, where one works in the setting of an exact category with duality. This gives us the presheaf of spectra on $\mathbf{S m} / S, X \mapsto K h(X)$, which is represented by a $\mathbb{P}^{1}$-spectrum KQ (see [13]). Just as $\mathrm{KGL}^{2 r, r}(X)$ is the "geometric" part $K_{0}(X)$ of $K$-theory, the classical Grothendieck group of vector bundles, $\mathrm{KQ}^{2 r, r}(X)$, has a description as a
Grothendieck-Witt group of quadratic forms. More precisely, one has the notion of a quadratic form in the derived category of perfect complexes on $X$, namely, a map $q: C^{*} \otimes_{\mathcal{O}_{X}}^{L} C^{*} \rightarrow O_{X}$ in $D_{\text {perf }}(X)$, which is symmetric with respect to the symmetry isomorphism $\tau: C^{*} \otimes_{\mathcal{O}_{X}}^{L} C^{*} \rightarrow$ $C^{*} \otimes_{\mathcal{O}_{X}}^{L} C^{*}$, and which is nondegenerate, in that the adjoint map to $q, C^{*} \rightarrow \operatorname{Hom}\left(C^{*}, \mathcal{O}_{X}\right)$, is an isomorphism in $D_{\text {perf }}(X)$. One can also introduce a shifted duality by using quadratic forms with values in $\mathcal{O}_{X}[r]$ for some $r$, and this, modulo a suitable derived isometry relation, gives the group $\mathrm{KQ}^{2 r, r}(X)$.

\section{Fundamental Invariants}

In many ways, the most fundamental motivic theory is the one represented by the unit, the motivic sphere spectrum $\mathbb{S}_{k}:=\Sigma_{\mathbb{P} 1}^{\infty}$ Spec $k_{+}$. This is the motivic analog of the classical sphere spectrum $\mathbb{S}:=\Sigma_{S^{1}}^{\infty} S^{0}$, which is the unit in $\mathrm{SH}$.

First a toy model, the derived category. For a commutative ring $R$, much of the basic structure of the derived category of $R$-modules, $D(R)$, follows from the structure of the (graded) endomorphism ring of the unit $R$ :

$$
\operatorname{Hom}_{D(R)}(R, R[n])= \begin{cases}R & \text { for } n=0, \\ 0 & \text { else. }\end{cases}
$$

For the classical stable homotopy category, things are much more mysterious: $\operatorname{Hom}_{\mathrm{SH}}\left(\mathbb{S}, \Sigma^{n} \mathbb{S}\right)$ is exactly the $n$th stable homotopy group $\pi_{n}(\mathbb{S})$. These have been intensively studied, but only the first fifty or so have been completely computed.

At least one does know that $\pi_{n}(\mathbb{S})=0$ for $n<0$, a reflection of the fact that $\pi_{m}\left(S^{n}\right)=0$ for $0<m<n$, and that $\pi_{0}(\mathbb{S})=\mathbb{Z}$, since $\pi_{n}\left(S^{n}\right)=H_{n}\left(S^{n}, \mathbb{Z}\right)=\mathbb{Z}$, this following from the connectivity of $S^{n}$ and the Hurewicz theorem. Additionally, Serre's finiteness theorem and the Freudenthal suspension theorem imply that $\pi_{n}(\mathbb{S})$ is a finite group for all $n>0$.

In the motivic setting life is already made more complicated by having a two-variable family of stable homotopy groups, $\pi_{a, b}\left(\mathbb{S}_{k}\right)(k):=\operatorname{Hom}_{\mathrm{SH}(k)}\left(\Sigma^{a, b} \mathbb{S}_{k}, \mathbb{S}_{k}\right)$ and homotopy sheaves $\pi_{a, b}\left(\mathbb{S}_{k}\right)$ on $\mathbf{S m} / k$.

Morel's homotopy $t$-structure $[11, \S 5.2]$ leads one to reindex these as $\pi_{n}\left(\mathbb{S}_{k}\right)_{m}:=\pi_{n-m,-m}\left(\mathbb{S}_{k}\right)$ and consider $\pi_{n}\left(\mathbb{S}_{k}\right)_{*}:=\bigoplus_{m} \pi_{n}\left(\mathbb{S}_{k}\right)_{m}$ as the motivic analog of $\pi_{n}(\mathbb{S})$.

For instance, Morel's stable $\mathbb{A}^{1}$-connectivity theorem [11, Theorem 4.2.10, Lemma 4.3.11] implies that $\pi_{n}\left(\mathbb{S}_{k}\right)_{*}=0$ for $n<0$. A beautiful analog of the identity $\pi_{0}(\mathbb{S})=\mathbb{Z}$ is Morel's computation of the motivic 0-stem [11, Theorem 6.4.1]

$$
\pi_{0}\left(\mathbb{S}_{k}\right)_{*}(k)=K_{*}^{M W}(k) .
$$

Here $K_{n}^{M W}(k)$ is the $n$th Milnor-Witt K-group of the field $k$, defined via explicit generators and relations by Hopkins and Morel [11, §6.3]. 
The unstable algebraic Hopf map is the map $\eta: A^{2} \backslash$ $\{0\} \rightarrow \mathbb{P}^{1}$ defining $\mathbb{P}^{1}$ as the quotient of $\mathbb{A}^{2} \backslash\{0\}$ modulo the $\mathbb{G}_{m}$-action, that is, $\eta((x, y))=[x: y]$. One has isomorphisms in $\mathcal{H} .(k),\left(\mathbb{A}^{2} \backslash\{0\},(1,0)\right) \cong S^{1} \wedge \mathbb{G}_{m} \wedge \mathbb{G}_{m}$, and $\mathbb{P}^{1} \cong$ $S^{1} \wedge \mathbb{G}_{m}$, so stably $\eta$ gives the class $\eta \in \pi_{0}\left(\mathbb{S}_{k}\right)_{-1}(k)$ and, by Morel's theorem, the corresponding element $\eta \in K_{-1}^{M W}(k)$.

The Milnor $K$-theory of $k, K_{*}^{M}(k)$, is simply the tensor algebra on the group of units $k^{\times}$, modulo the ideal generated by $\{a \otimes(1-a) \mid a \in k \backslash\{0,1\}\}$. There is a surjective ring homomorphism $K_{*}^{M W}(k) \rightarrow K_{*}^{M}(k)$ with kernel the two-sided ideal generated by $\eta$. In fact, it follows from the very definition that $K_{*}^{M W}(k)$ is generated as a graded ring by $\eta \in K_{-1}^{M W}(k)$ and elements $[u] \in K_{1}^{M W}(k)$ for $u \in k^{\times}$, corresponding via Morel's theorem to $u$ viewed as a map Spec $k_{+} \rightarrow \mathbb{G}_{m}$, with the following relations [11, Definition 6.3.1]:

1. $\eta \cdot[u]=[u] \cdot \eta$ for all $u \in k \times$.

2. $[u] \cdot[1-u]=0$ for $u \in k \backslash\{0,1\}$.

3. $[u v]=[u]+[v]+\eta[u][v]$ for $u, v \in k^{\times}$.

4. $\eta \cdot(2+\eta[-1])=0$.

The $\operatorname{map} K_{*}^{M W}(k) \rightarrow K_{*}^{M}(k)$ is defined by sending $\eta$ to zero and $[u]$ to the class $\{u\}$ of $u$.

The assignment of a field $F$ to the Milnor-Witt ring $K_{*}^{M W}(F)$ or the Milnor $K$-theory ring $K_{*}^{M}(F)$ both extend to Nisnevich sheaves $\mathcal{K}_{*}^{M W}, \mathcal{K}_{*}^{M}$ on $\mathrm{Sm} / k$, and Morel's isomorphism extends to an isomorphism of sheaves

$$
\pi_{0}\left(\mathbb{S}_{k}\right)_{*} \cong \mathcal{K}_{*}^{M W} .
$$

The Milnor-Witt ring thus combines the Milnor $K$ groups $K_{*}^{M}(k)$ with information having to do with quadratic forms. $K_{0}^{M W}(k)$ is exactly the Grothendieck-Witt ring $\mathrm{GW}(k)$ of nondegenerate symmetric bilinear forms over $k$, and for $n<0, K_{n}^{M W}(k)$ is the Witt ring, that is, $\mathrm{GW}(k)$ modulo the hyperbolic form. Explicitly, the rank one form $q_{u}(x)=u x^{2}, u \in k^{\times}$, gets sent to $\langle u\rangle:=1+\eta[u] \in$ $K_{0}^{M W}(k)$. The relation (3) translates into the multiplicativity $\langle u v\rangle=\langle u\rangle \cdot\langle v\rangle$, which the relation $q_{u} \cdot q_{v}=q_{u v}$ in $\mathrm{GW}(k)$ requires. The hyperbolic form $h(x, y)=x^{2}-y^{2}$ gets sent to $2+\eta[-1]$ and is thus $\eta^{n} \cdot h=0$ in $K_{-n}^{M W}(k)$ for all $n>0$. This shows that the map GW $(k) \rightarrow K_{0}^{M W}(k)$ descends to $W(k) \rightarrow K_{-n}^{M W}(k)$ for all $n>0$. Morel shows that all these maps are isomorphisms [11, Lemmas 6.3.8 and 6.3.9].

For $n>0$, there is an exact sequence

$$
0 \rightarrow I^{n+1} \rightarrow K_{n}^{M W}(k) \rightarrow K^{M}(k) \rightarrow 0,
$$

where $I \subset \mathrm{GW}(k)$ is the augmentation ideal of quadratic forms of virtual rank zero [11, Theorem 6.4.5]. All this extends to the sheaf setting without change; in particular $\mathcal{K}_{0}^{M W}$ is the sheaf $\mathcal{G} \mathcal{W}$ of Grothendieck-Witt rings on $\mathrm{Sm} / k$.

One can try to understand the sheaves $\pi_{*}\left(\mathbb{S}_{k}\right)_{*}$ via unit maps to various motivic ring spectra. For the oriented theories $\mathcal{E}=M \mathbb{Z}, \mathrm{KGL}, \mathrm{MGL}, \eta$ acts as zero, $\pi_{0}(\varepsilon)_{*}=$ $\mathcal{K}_{*}^{M}$, and the respective unit map induces the surjection $\mathcal{K}_{*}^{M W} \rightarrow \mathcal{K}_{*}^{M}$. As a first attempt, consider the unit map $u_{\mathrm{KGL}}: \mathbb{S}_{k} \rightarrow \mathrm{KGL}$. For $*=0$, this is simply the rank map $\mathcal{G W} \rightarrow \mathbb{Z}$, so these theories do not carry any of the quadratic forms information in $\mathcal{K}_{*}^{M W}$.

Hermitian $K$-theory gives a better approximation to the sphere spectrum than $M \mathbb{Z}, \mathrm{KGL}$, or MGL. In fact the unit map $\mathbb{S}_{k} \rightarrow \mathrm{KQ}$ induces an isomorphism on $\pi_{0}(-)_{*}$ for $* \leq 3$ by work of Suslin and Asok-Fasel. This is perhaps not so surprising, at least for $\pi_{0}(-)_{0}$ as $\pi_{0}\left(\mathbb{S}_{k}\right)_{0}=\mathcal{G W}=$ $\mathrm{KQ}^{0,0}$, the first identity from Morel's theorem and the second more or less by construction. In her 2019 doctoral thesis, Maria Yakerson [20] considers $\pi_{0}(\mathrm{MSL})_{*}$ and shows by a direct computation that this is also $\mathcal{K}_{*}^{M W}$.

Relying heavily on properties of KQ, RöndigsSpitzweck-Østvær [15] compute the motivic stable 1-stem. Here is the result; for simplicity we give the statement only in characteristic zero, although suitably modified, it remains true in arbitrary characteristic $\neq 2$.

Theorem 1 ([15, Theorem 5.5]). Let $k$ be a field characteristic 0 . The unit map $\mathbb{S}_{k} \rightarrow \mathrm{KQ}$ induces a short exact sequence of Nisnevich sheaves

$$
0 \rightarrow \mathcal{K}_{2+n}^{M} / 24 \rightarrow \pi_{1}\left(\mathbb{S}_{k}\right)_{n} \rightarrow \pi_{1}\left(f_{0} \mathrm{KQ}\right)_{n} .
$$

The right-hand map is surjective for $n \leq 4$. In addition, $\pi_{1}\left(f_{0} \mathrm{KQ}\right)_{n}=0$ for $n \leq-2$; in particular $\pi_{1}\left(\mathbb{S}_{k}\right)_{-2} \cong \mathbb{Z} / 24$ and $\pi_{1}\left(\mathbb{S}_{k}\right)_{n}=0$ for $n<-2$.

Here $f_{0} \mathrm{KQ}$ is the so-called effective cover of KQ. This involves Voevodsky's slice filtration, a motivic analog of the classical Moore-Postnikov tower. The cover $f_{0} \mathrm{KQ} \rightarrow \mathrm{KQ}$ induces an isomorphism on $\pi_{i}(-)_{n}$ for all $n \leq 0$, so in that range, one can replace $f_{0} \mathrm{KQ}$ with hermitian $K$-theory KQ itself. We refer the reader to the excellent survey article [7] as well as the original paper [15] for details on the theorem, the slice filtration, and many other interesting topics.

A remarkable and perhaps unexpected application of the motivic theory has been to give a new and quite effective tool for computing classical stable homotopy groups of spheres. The (topological) Adams and Adams-Novikov spectral sequences promote higher multiplicative structures in singular cohomology or complex cobordism to yield computations of the stable homotopy groups of spheres. Computations of the differentials are often very difficult.

There are motivic analogs of these spectral sequences, using the motivic cohomology spectrum or the algebraic cobordism spectrum as motivic replacements for singular cohomology and complex cobordism. The motivic versions involve an additional grading, the Tate twist, which tends to split the classical differentials into a number of summands. This additional information is often very helpful in resolving ambiguities and determining the 
differentials in the classical spectral sequence. Using this approach in a number of papers, Isaksen, $\mathrm{Xu}$, and Wang (see for example [6], [19]) have corrected earlier computations and have begun pushing beyond; their paper on the 61st stem has the beautiful geometric consequence: the 61-sphere has a unique smooth structure, and it is the last odd-dimensional case. Pushing up to $\pi_{126}$ could resolve the last remaining case of the Kervaire invariant one conjecture. We refer the reader to [7] for a much more complete survey of this fascinating topic, as well as many other results on the motivic homotopy groups of spheres.

\section{Plus and Minus}

One fascinating aspect of motivic homotopy theory is how it unifies two different homotopical worlds via complex and real embeddings. An algebraic variety $X$ over $\mathbb{R}$ determines simultaneously two topological spaces, namely, the space of $\mathbb{C}$-points $X(\mathbb{C})$ and the space of $\mathbb{R}$-points $X(\mathbb{R})$, with the topology induced by $\mathbb{C}, \mathbb{R}$, respectively. As a simple example, the algebraic projective line $\mathbb{P}^{1}$ has $\mathbb{P}^{1}(\mathbb{C}) \cong$ $S^{2}$ and $\mathbb{P}^{1}(\mathbb{R}) \cong S^{1}$. This dual nature extends to realization functors $\operatorname{Re}_{\mathbb{C}}: \mathrm{SH}(\mathbb{C}) \rightarrow \mathrm{SH}, \mathrm{Re}_{\mathbb{R}}: \mathrm{SH}(\mathbb{R}) \rightarrow \mathrm{SH}$. Morel has introduced the heuristic principle, that a conjecture in $\mathrm{SH}(k)$ can be tested by seeing if it remains true in $\mathrm{SH}$ after applying $\mathrm{Re}_{\mathbb{C}}$ and $\mathrm{Re}_{\mathbb{R}}$ for all complex or real embeddings $k \rightarrow \mathbb{C}, k \rightarrow \mathbb{R}$.

As an elementary but fundamental example, consider the symmetry isomorphism $\tau: \mathbb{P}^{1} \wedge \mathbb{P}^{1} \rightarrow \mathbb{P}^{1} \wedge \mathbb{P}^{1}$, exchanging the factors; clearly $\tau^{2}=\mathrm{Id}$. Under the complex realization, this is $S^{4}=S^{2} \wedge S^{2} \rightarrow S^{2} \wedge S^{2}=S^{4}$, which is homotopic to the identity map, while under the real realization, this is $S^{2}=S^{1} \wedge S^{1} \rightarrow S^{1} \wedge S^{1}=S^{2}$, which is homotopic to minus the identity map. Thus, the map $\tau$ gives a way of distinguishing between $\mathrm{Re}_{\mathbb{C}}$ and $\mathrm{Re}_{\mathbb{R}}$, even after passing to the stable setting. We can use the additive nature of $\mathrm{SH}(k)$ to form the two idempotent endomorphisms of the sphere spectrum $\mathbb{S}_{k}$ (after inverting 2),

$$
\tau_{+}:=\frac{1+\tau}{2}, \quad \tau_{-}:=\frac{1-\tau}{2} .
$$

This splits $\mathbb{S}_{k}$ in $\mathrm{SH}(k)[1 / 2]$ as

$$
\mathbb{S}_{k}=\mathbb{S}_{k}^{+} \oplus \mathbb{S}_{k}^{-},
$$

with $\tau_{+}$acting as the identity on $\mathbb{S}_{k}^{+}$and as zero on $\mathbb{S}_{k}^{-}$, and with $\tau_{-}$having the opposite behavior. Since $\mathbb{S}_{k}$ is the unit for the monoidal structure on $\mathrm{SH}(k)$, this splitting of $\mathbb{S}_{k}$ into plus and minus factors splits the whole category $\mathrm{SH}(k)[1 / 2]$ as the product of two tensor triangulated categories

$$
\mathrm{SH}(k)[1 / 2]=\mathrm{SH}(k)_{+} \times \mathrm{SH}(k)_{-} .
$$

The fact that $\tau$ induces Id under $\operatorname{Re}_{\mathbb{C}}$ and $-\mathrm{Id}$ under $\operatorname{Re}_{\mathbb{R}}$ says that $\mathrm{Re}_{\mathbb{C}}$ factors through the projection to $\mathrm{SH}(k)_{+}$and $\mathrm{Re}_{\mathbb{R}}$ factors through the projection to $\mathrm{SH}(k)_{-}$after inverting 2 .
The two factors $\mathrm{SH}(k)_{+}$and $\mathrm{SH}(k)_{-}$behave quite differently. One aspect of this is the relation with Voevodsky's triangulated category of motives, $\operatorname{DM}(k)$. There is a motivic Eilenberg-MacLane functor $\mathrm{EM}_{\text {mot }}: \mathrm{DM}(k) \rightarrow \mathrm{SH}(k)$ that formally mimics the classical one EM $: D(\mathbf{A b}) \rightarrow \mathrm{SH}$. The fact that the higher stable homotopy groups of the sphere spectrum $\mathbb{S}$ in $\mathrm{SH}$ are all torsion implies that the classical Eilenberg-MacLane functor is an equivalence after $\mathbb{Q}$-localization. As the symmetry operator $\tau_{\mathbb{Z}}: \mathbb{Z}(1) \otimes$ $\mathbb{Z}(1) \rightarrow \mathbb{Z}(1) \otimes \mathbb{Z}(1)$ is the identity, $\mathrm{EM}_{\text {mot }}$ factors through $\mathrm{SH}(k)_{+}$(after inverting 2). Thus, for fields $k$ such that

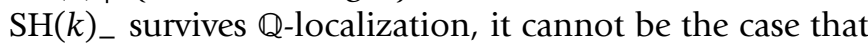
$\mathrm{EM}_{\text {mot }}$ is a $\mathbb{Q}$-equivalence. On the other hand, a result of Cisinski-Déglisé [4, Theorems 16.1.4 and 16.2.13] tells us that $\mathrm{EM}_{\text {motQ }}: \mathrm{DM}(k)_{\mathbb{Q}} \rightarrow \mathrm{SH}(k)_{+\mathbb{Q}}$ is an equivalence, so $\mathrm{EM}_{\text {mot }}: \mathrm{DM}(k)_{\mathbb{Q}} \rightarrow \mathrm{SH}(k)_{\mathbb{Q}}$ is an equivalence exactly when $\operatorname{SH}(k)_{-\mathbb{Q}}=0$.

For which fields $k$ does $\mathrm{SH}(k)$ _ survive $\mathbb{Q}$-localization? Again, the answer to this lives in $\operatorname{End}\left(\mathbb{S}_{k}\right)=\mathrm{GW}(k)$. The unit in $\operatorname{DM}(k)$ is the weight-zero Tate motive $\mathbb{Z}(0)$, and it is not hard to show that

$$
\operatorname{End}_{\mathrm{DM}(k)}(\mathbb{Z}(0))=\mathbb{Z} \cdot \operatorname{Id}_{\mathbb{Z}(0)} \cdot
$$

Now, $\mathrm{SH}(k)_{-}$survives $\mathbb{Q}$-localization if and only if $\operatorname{End}_{\mathrm{SH}(k)}\left(\mathbb{S}_{-}\right)_{\mathbb{Q}} \neq\{0\}$. Also, as the map $\operatorname{End}_{\mathrm{SH}(k)}\left(\mathbb{S}_{k}\right) \rightarrow$ $\operatorname{End}_{\mathrm{DM}(k)}(\mathbb{Z}(0))$ induced by the adjoint to $\mathrm{EM}_{\text {mot }}$ is the rank homomorphism $\mathrm{GW}(k) \rightarrow \mathbb{Z}$, we see that $\mathrm{SH}(k)_{-}$survives $\mathbb{Q}$-localization if and only if the augmentation ideal $I:=\operatorname{ker}(\mathrm{rnk}) \subset \mathrm{GW}(k)$ is nontorsion. This property of $k$ has been studied classically, and the answer is: $I$ is not a torsion group if and only if $k$ admits an ordering or, equivalently, -1 is not a sum of squares in $k$. In particular, $\mathrm{SH}(k)$ _ survives $\mathbb{Q}$-localization for any field $k$ that admits an embedding into $\mathbb{R}$.

To summarize: the motivic stable homotopy category $\mathrm{SH}(k)$ splits into two pieces after inverting 2, the plus part and the minus part. Rationally, the plus part is the same as Voevodsky's triangulated category of motives, a motivic reflection of the fact that the classical stable homotopy category is rationally the same as the derived category of abelian groups. The minus part survives $\mathbb{Q}$-localization exactly when -1 is not a sum of squares in $k$, for example, if $k$ admits an embedding into $\mathbb{R}$.

This suggests that the mysterious minus part could be approached via the real realization for each embedding $k \hookrightarrow \mathbb{R}$. As a simple example of Morel's heuristic, look back at our friend the involution $\tau: \mathbb{P}^{1} \wedge \mathbb{P}^{1} \rightarrow \mathbb{P}^{1} \wedge \mathbb{P}^{1}$. This induces an endomorphism of $\mathbb{S}_{k}$, giving us an element $[\tau]$ of $\mathrm{GW}(k)$. What element is this? We know under complex realization $\mathbb{P}^{1}$ becomes an $S^{2}$, so under $\mathrm{GW}(k) \rightarrow \mathrm{GW}(\mathbb{C})=$ $\mathbb{Z}$ (which is the rank map) $[\tau]$ maps to 1 . One can show that the map $\mathrm{GW}(k) \rightarrow \operatorname{End}_{\mathrm{SH}}(\mathbb{S})=\mathbb{Z}$ under real realization is the signature map $\mathrm{GW}(k) \rightarrow \mathrm{GW}(\mathbb{R}) \stackrel{\text { sig }}{\longrightarrow} \mathbb{Z}$. As $\mathbb{P}^{1}$ 
becomes an $S^{1}$ under real realization, $[\tau]$ maps to -1 , so $[\tau]$ has signature -1 . In fact, if we expand our horizons a bit and allow ourselves to work in the motivic stable homotopy category over $\mathbb{Z}$, then as $\tau$ is defined over $\mathbb{Z}$, we should expect that $[\tau]$ lives in $\mathrm{GW}(\mathbb{Z}) \subset \mathrm{GW}(\mathbb{Q})$. $\mathrm{GW}(\mathbb{Z})$ is quite simple; in fact the map $\mathbb{Z} \rightarrow \mathbb{R}$ induces an isomorphism $\mathrm{GW}(\mathbb{Z}) \rightarrow \mathrm{GW}(\mathbb{R})$, and the pair (rnk, sig) : $\mathrm{GW}(\mathbb{R}) \rightarrow \mathbb{Z} \times \mathbb{Z}$ is an injective ring homomorphism. Thus the information $\operatorname{rnk}[\tau]=1, \operatorname{sig}[\tau]=-1$ would completely determine $[\tau]$ and tells us that $[\tau]$ is the one-dimensional form $x \mapsto-x^{2}$.

We underscore that the heuristic reasoning given above is not a proof. Although one suspects that $\operatorname{End}_{\mathrm{SH}(\mathbb{Z})}\left(\mathbb{S}_{\mathbb{Z}}\right)=\mathrm{GW}(\mathbb{Z})$, this has not been proven, and there are in fact quite fundamental problems that stand in the way. Even showing that the image of $\operatorname{End}_{\mathrm{SH}(\mathbb{Z})}\left(\mathbb{S}_{\mathbb{Z}}\right)$ in $\operatorname{End}_{\mathrm{SH}(\mathbb{Q})}\left(\mathbb{S}_{\mathbb{Q}}\right)=\mathrm{GW}(\mathbb{Q})$ is the subring $\mathrm{GW}(\mathbb{Z})$ is still out of reach, but perhaps not as far as the complete computation of $\operatorname{End}_{\mathrm{SH}(\mathbb{Z})}\left(\mathbb{S}_{\mathbb{Z}}\right)$. The computation of the image of $\operatorname{End}_{\mathrm{SH}(\mathbb{Z})}\left(\mathbb{S}_{\mathbb{Z}}\right)$ in $\operatorname{End}_{\mathrm{SH}(\mathbb{Q})}\left(\mathbb{S}_{\mathbb{Q}}\right)$ relies on extending the known theory of hermitian $K$-theory to schemes over $\mathbb{Z}[1 / 2]$ to schemes over $\mathbb{Z}$. With our present knowledge, the best we can say is that the image of $\operatorname{End}_{\mathrm{SH}(\mathbb{Z})}\left(\mathbb{S}_{\mathbb{Z}}\right)$ in $\operatorname{End}_{\mathrm{SH}(\mathbb{Q})}\left(\mathbb{S}_{\mathbb{Q}}\right)$ is contained in $\mathrm{GW}(\mathbb{Z}[1 / 2]) \subset \mathrm{GW}(\mathbb{Q})$. As $\mathrm{GW}(\mathbb{Z}[1 / 2])$ is only a little larger than $\mathrm{GW}(\mathbb{Z})$ (there is an additional 2-torsion element), this is not too bad, but still it would be nice to get all the way down to $\mathrm{GW}(\mathbb{Z})=$ $\mathrm{GW}(\mathbb{R})$.

On the positive side, Bachmann's results (see Theorems 2 and 3 below) can be viewed as making at least the real part of this heuristic precise. In any case, Morel has verified the above guess for $[\tau]$, showing [11, Remark 6.3.5] that $[\tau]=\langle-1\rangle=1+\eta[-1] \in K_{0}^{M W}(k)=\mathrm{GW}(k)$.

\section{The Algebraic Hopf Map}

Another way to get at the mysterious minus part of $\mathrm{SH}(k)$ is by inverting the algebraic Hopf map. In topology, the Hopf map $\eta^{\text {top }}: S^{3} \rightarrow S^{2}$ is the first "surprising" bit of the homotopy groups of spheres. $\eta^{\text {top }}$ is the generator of $\pi_{3}\left(\mathbb{S}^{2}\right) \cong \mathbb{Z}$, and its first suspension $\Sigma \eta^{\text {top }}$ is also a generator of $\pi_{4}\left(S^{3}\right)=\mathbb{Z} / 2$. The Freudenthal suspension theorem shows that further suspension gives isomorphisms $\pi_{4}\left(S^{3}\right) \cong \pi_{n+1}\left(S^{n}\right), n \geq 3$, so stably $\eta^{t o p}$ generates $\pi_{1}(\mathbb{S})=\operatorname{colim}_{n} \pi_{n+1}\left(S^{n}\right) \cong \mathbb{Z} / 2$. We can view $S^{3}$ as the unit sphere in $\mathbb{C}^{2}$, which shows that our algebraic Hopf map $\eta: \mathbb{A}^{2} \backslash\{0\} \rightarrow \mathbb{P}^{1}$ has $\mathbb{C}$-realization (for $k \subset \mathbb{C}$ ) equal to $\eta^{\text {top }}$. What is the real realization? $\mathbb{A}^{2} \backslash\{0\}$ becomes $\mathbb{R}^{2} \backslash\{0\}$, and $\mathbb{P}^{1}$ becomes $\mathbb{R} \mathbb{P}^{1} \cong S^{1}$. The inclusion of the unit circle defines a homotopy equivalence $S^{1} \sim \mathbb{R}^{2} \backslash\{0\}$, and $\eta$ realizes the map $\times 2: S^{1} \rightarrow S^{1}$, giving the homotopy class $\left[\operatorname{Re}_{\mathbb{R}}(\eta)\right] \in \pi_{1}\left(S^{1}\right)=\pi_{0}(\mathbb{S})=\mathbb{Z}$, i.e., $\left[\operatorname{Re}_{\mathbb{R}}(\eta)\right]=$ $2 \in \mathbb{Z}$. Note that in contrast to $\left[\operatorname{Re}_{\mathbb{C}}(\eta)\right] \in \pi_{1}(\mathbb{S})=\mathbb{Z} / 2$, $\left[\operatorname{Re}_{\mathbb{R}}(\eta)\right] \in \pi_{0}(\mathbb{S})$ is a nontorsion element.

If we formally invert $\eta$ in $\operatorname{SH}(k)$, then under $\mathbb{C}$ - realization we have inverted a 2-torsion element, so $\mathbb{C}$-realization sends $\mathrm{SH}(k)\left[\eta^{-1}\right]$ to zero, while $\mathbb{R}$-realization merely inverts 2 . This suggests that $\mathrm{SH}(k)\left[\eta^{-1}, 1 / 2\right] \cong \mathrm{SH}(k)_{-}$, which is in fact the case. This identity follows from a basic relation in $\mathrm{GW}(k)$.

As mentioned above, Morel has shown that $[\tau]=$ $\langle-1\rangle=1+\eta[-1]$. Splitting $\mathrm{SH}(k)[1 / 2]$ into plus and minus parts corresponds to the two idempotents $\tau_{+}:=(1+[\tau]) / 2$, $\tau_{-}:=(1-[\tau]) / 2$, with $\tau_{ \pm}$acting by the identity on $\mathrm{SH}(k)_{ \pm}$ and by zero on $\mathrm{SH}(k)_{\mp}$. As $\tau_{-}=(-1 / 2) \eta[-1]$, both $\eta$ and $[-1]$ are invertible on $\mathrm{SH}(k)_{-}$. Similarly, as $\tau_{+}=\mathrm{Id}+$ $(1 / 2) \eta[-1], \eta[-1]=0$ on $\mathrm{SH}(k)_{+}$. In fact, $\mathrm{SH}(k)_{+}\left[\eta^{-1}\right]=0$. As we have already seen, $\eta^{n} \cdot K_{0}^{M W}(k)=K_{-n}^{M W}(k)=W(k)$ for all $n>0$, and thus

$$
K_{*}^{M W}(k)\left[\eta^{-1}\right]=\operatorname{colim}_{n, \times \eta} K_{-n}^{M W}(k)=W(k) .
$$

The augmentation ideal $I \subset \mathrm{GW}(k)$ is also an ideal in $W(k)$, and $W(k) / I=\mathbb{Z} / 2$; and as the projection onto the plus part gives the rank map $\mathrm{GW}(k)[1 / 2] \rightarrow$ $\mathbb{Z}[1 / 2]$, we see that the projection of $\operatorname{End}_{\mathrm{SH}(k)}\left(\mathbb{S}_{k}\right)\left[\eta^{-1}, 1 / 2\right]$ to $\operatorname{End}_{\mathrm{SH}(k)_{+}}\left(\mathbb{S}_{k}^{+}\right)\left[\eta^{-1}, 1 / 2\right]$ is the zero map, and thus $\mathrm{SH}(k)_{+}\left[\eta^{-1}\right]=0$.

After inverting 2, inverting $[-1]$ has exactly the same effect as inverting $\eta$. Let $\rho: S_{k}^{0} \rightarrow \mathbb{G}_{m}$ be the map corresponding to $-1 \in \mathbb{G}_{m}(k)$. We also use $\rho: \mathbb{S}_{k} \rightarrow \mathbb{G}_{m} \wedge \mathbb{S}_{k}$ for the stable version, corresponding to $[-1] \in K_{1}^{M W}(k)$. Then $\mathrm{SH}(k)_{-}\left[\rho^{-1}\right]=\mathrm{SH}(k)_{-}$and $\mathrm{SH}(k)_{+}\left[\rho^{-1}\right]=0$. We have seen the interpretation of $\eta$ as the classical Hopf map via the complex realization and as multiplication by 2 on $S^{1}$ via the real realization. We can do the same for the map $\rho$. The complex realization of $\mathbb{G}_{m}$ is $\mathbb{C}^{\times} \sim S^{1}$, and $\operatorname{Re}_{\mathbb{C}}([-1])$ is the inclusion of $S^{0}$ to $S^{1}$ as $\{ \pm 1\}$. As this map is clearly homotopic to 0 , inverting $\operatorname{Re}_{\mathbb{C}}(\rho)$ will kill the entire stable homotopy category. The real realization of $\rho$ is $S^{0} \rightarrow \mathbb{R}^{\times}$, again being the inclusion of $\{ \pm 1\}$ into $\mathbb{R}^{\times}$, but this time the map is a homotopy equivalence, even without inverting 2. This suggests that $\mathrm{SH}(k)\left[\rho^{-1}\right]$ should be closely related to the classical stable homotopy category via the real realization.

\section{The $\rho$-inverted Motivic Stable Homotopy Category}

Bachmann has proven a much more precise statement about the $\rho$-localization of $\mathrm{SH}(k)$. We first give the simple version.

Theorem 2 (Bachmann [3]). The real realization $\mathrm{Re}_{\mathbb{R}}$ : $\mathrm{SH}(\mathbb{R}) \rightarrow \mathrm{SH}$ induces an equivalence $\mathrm{SH}(\mathbb{R})\left[\rho^{-1}\right] \rightarrow \mathrm{SH}$.

This is just a special case. In general, Bachmann considers the motivic stable homotopy category over a basescheme $X$. One has the topological space of "real" points of $X, \mathcal{R}(X)$. Here, the points of $\mathcal{R}(X)$ are pairs $(x, \alpha)$ consisting of a point $x$ of $X$ and an ordering $\alpha$ on the residue field $k(x)$. The topology is formed by incorporating the 
orderings and the Zariski topology on $X$. As any topological space, $\mathcal{R}(X)$ has a homotopy theory of sheaves of simplicial sets, giving rise to a stable homotopy category $\mathrm{SH}(\mathcal{R}(X))$. Bachmann's general theorem is

Theorem 3. There is an equivalence of $\mathrm{SH}(X)\left[\rho^{-1}\right]$ with $\mathrm{SH}(\mathcal{R}(X))$.

For $X=\operatorname{Spec} \mathbb{R}$, there is a unique ordering on the residue field $\mathbb{R}$ of the unique point of $X, \operatorname{sosH}(\mathcal{R}(\operatorname{Spec} \mathbb{R})$ ) is just the usual stable homotopy category $\mathrm{SH}$, giving back the special case Theorem 2.

In particular, Bachmann's theorem gives a canonical object in $\mathrm{SH}(\mathbb{R})\left[\rho^{-1}\right]$ with real realization MU. Recognizing the important role that complex cobordism plays in understanding the structure of $\mathrm{SH}$, it would be interesting to see to what extent one can lift this object to $\mathrm{SH}(\mathbb{Q})$ and how unique such a lifting would be. For lifting to $\mathrm{SH}(\mathbb{R})$, an obvious candidate would be the cobordism spectrum built out of the restriction of scalars $R_{\mathbb{C} / \mathbb{R}} \mathrm{GL}_{n}, n=0,1,2, \ldots$, but the general picture is unclear.

As we have seen, $\mathrm{SH}(k)\left[\rho^{-1}\right]$ and $\mathrm{SH}(k)\left[\eta^{-1}\right]$ both agree with $\mathrm{SH}(k)_{-}$after inverting 2, so Bachmann's theorem gives a workable description of $\mathrm{SH}(k)_{-}$, with some quite beautiful consequences. One of these is a new proof of a result of Ananyevskiy-Levine-Panin [1], an analog of Serre's theorem on the finiteness of $\pi_{n}(\mathbb{S})$ for $n>0$.

Theorem 4. The homotopy sheaves $\pi_{i}\left(\mathbb{S}_{k}^{-}\right)_{*}$ are torsion for all $i \neq 0$.

Combining the Cisinski-Déglisé result identifying $\operatorname{DM}(k)_{\mathbb{Q}}$ with $\mathrm{SH}(k)_{+\mathbb{Q}}$, Morel's $\mathbb{A}^{1}$-connectivity theorem $\left(\pi_{i}\left(\mathbb{S}_{k}\right)_{*}=0\right.$ for $\left.i<0\right)$, and Morel's theorem $\pi_{0}\left(\mathbb{S}_{k}\right)_{*}=$ $K_{*}^{M W}$, we thus have a complete description of $\pi_{*}\left(\mathbb{S}_{k}\right)_{* \mathbb{Q}}$ in terms of the Witt sheaves $\mathcal{W}$ and motivic cohomology sheaves $\mathcal{H}^{p, q}$.

One sees the complexity of motivic homotopy theory as compared to the classical case, as well as an interesting parallel, in the following tables:

$$
\begin{array}{lll}
\pi_{i}(\mathbb{S})=0 \quad \text { for } i<0, & \pi_{i}\left(\mathbb{S}_{k}\right)_{*}=0 \quad \text { for } i<0, \\
\pi_{0}(\mathbb{S})=\mathbb{Z}, & \pi_{0}\left(\mathbb{S}_{k}\right)_{*}=\mathcal{K}_{*}^{M W}, \\
\pi_{i}(\mathbb{S})_{\mathbb{Q}}=0 \quad \text { for } i>0, & \pi_{i}(\mathbb{S})_{n \mathbb{Q}}=\mathcal{H}_{\mathbb{Q}}^{n-i, n} \quad \text { for } i>0 .
\end{array}
$$

If we think of $\mathcal{H}^{n-i, n}$ as the "cohomology of a point," we see another way in which the motivic theory differs from the classical one: a point (that is, the spectrum of a field) can have nontrivial higher cohomology in the motivic case.

To finish off the story, we note that $\mathcal{H}_{\mathbb{Q}}^{p, q}=0$ for $q<0$, $\mathcal{H}^{p, 0}=0$ for $p \neq 0$, and $\mathcal{H}^{0,0}=\mathbb{Z}$. The Beilinson-Soulé conjectures assert that the sheaf $\mathcal{H}_{\mathbb{Q}}^{p, q}$ is zero for $p \leq 0$ and $q>0$, but this is known only for $q=1$. Thus, in addition to Morel's vanishing $\left(\pi_{i}\left(\mathbb{S}_{k}\right)_{*}=0\right.$ for $\left.i<0\right)$, we have $\pi_{i}(\mathbb{S})_{n \mathbb{Q}}=0$ for $i>0$ and $n \leq 0$, and the Beilinson-Soule conjectures imply the additional vanishing $\pi_{i}(\mathbb{S})_{n \mathbb{Q}}=0$ for $n>0$ and $i \geq n$.

\section{Cohomology Theories, Orientations, and Characteristic Classes}

Let $\mathcal{E}$ be a $G$-oriented theory ( $G=\mathrm{GL}, \mathrm{SL}, \mathrm{Sp}$ ) and let $V \rightarrow$ $X$ be a $G$-vector bundle of rank $r$. Via the Thom isomorphism $\mathcal{E}^{a, b}(X) \rightarrow \mathcal{E}^{2 r+a, r+b}(\operatorname{Th}(V))$, the unit $1_{X} \in \mathcal{E}^{0,0}(X)$ gives the Thom class $\operatorname{th}(V) \in \mathcal{E}^{2 r, r}(\operatorname{Th}(V))$. The Euler class $e(V) \in \mathcal{E}^{2 r, r}(X)$ is the pullback of th $(V)$ by the zero-section. Starting with the Euler class, there are often ways of constructing other quite useful characteristic classes for vector bundles.

The theory of Chern classes lies at the very roots of our modern theory of motives. Grothendieck showed how to make Chern's topological theory algebraic, yielding a theory of Chern classes of algebraic vector bundles with values in the Chow ring. His approach relies on the existence of a first Chern class for line bundles and the projective bundle formula, which for a vector bundle $E \rightarrow X$ expresses the Chow ring of a projective space bundle $\mathbb{P}(E) \rightarrow X$ as a free module over the Chow ring of $X$, with generator powers of the first Chern class of the tautological line bundle. In fact, Grothendieck worked in the abstract setting, using a contravariant functor $A^{*}$ from smooth varieties to graded rings, satisfying certain axioms. A version of these axioms is satisfied (suitably modified for bi-graded theories) for the functors $X \mapsto \mathcal{E}^{* *}(X)$, where $\mathcal{E}^{* *}$ is an oriented commutative ring spectrum in the sense indicated in "Some Useful Motivic Cohomology Theories," and are all compatible via the complex realization functor with the corresponding topological theory of characteristic classes for $\operatorname{Re}_{\mathbb{C}}(\mathcal{E})$. The Euler class construction is recovered as the top Chern class, in particular, for a line bundle $L, e(L)=c_{1}(L)$, so one can use the Euler class as the starting point for the whole construction.

For example, one has a motivic theory of Conner-Floyd Chern classes for MGL, which under $\mathbb{C}$-realization maps to the classical Conner-Floyd Chern classes for MU. In fact, once one has set up the machinery, the motivic setting for oriented theories is formally exactly the same as the topological setting for complex oriented theories. This is made more precise by the universality of MGL as a motivicoriented theory, proved by Panin-Pimenov-Röndigs [12], exactly parallel to the universality of MU among complex oriented theories, and by the fact that the coefficient ring $\mathrm{MGL}^{2 * * *}(k)$ is equal to the coefficient ring of MU, namely, the Lazard ring, classifying rank one commutative formal group laws. This fundamental result was first stated by Hopkins-Morel and was given a complete proof by Hoyois [5]. In positive characteristics, one needs to invert the characteristic for the proof, although it is conjectured that the result remains true integrally. 
Complex cobordism and the theory of formal group laws give rise to some of the most basic theorems on the structure of the stable homotopy category, for instance, the nilpotence theorem of Devinatz-Hopkins-Smith. One might expect that via the close connection of MGL with $\mathrm{MU}$, one could lift these results to $\mathrm{SH}(k)$. However, the algebraic Hopf map $\eta$ acts as the zero map on $\mathrm{MGL}^{* *}$, so $\mathrm{MGL}^{* *}$ dies after inverting $\eta$, and one cannot use the theory of oriented spectra to say anything much about $\mathrm{SH}(k)\left[\eta^{-1}\right]$ or $\mathrm{SH}(k)_{-}$. However, theories with SL or Sp orientations do not automatically die after inverting $\eta$, and these will thus be useful for understanding $\mathrm{SH}(k)_{-}$.

Panin-Walter [14] have considered the symplectically oriented theories as motivic analogs of the classical quaternionic theories. They have constructed a motivic analog of quaternionic projective space as the symplectic Grassmannian $\mathbb{A} \mathbb{P}^{n}$, this being the open subscheme of the usual Grassmann variety of 2 -planes in $2 n+2$-space, $\operatorname{Gr}(2,2 n+2)$, consisting of those 2-planes for which the standard symplectic form $\omega_{2 n+2}$ on $A^{2 n+2}$ is nondegenerate, and thus the restriction $\tilde{E}_{2} \rightarrow \mathbb{H} \mathbb{P}^{n}$ of the tautological 2-plane bundle on $\operatorname{Gr}(2,2 n+2)$ is a symplectic vector bundle.

This generalizes to the setting of a rank $2 n+2$ symplectic bundle $\left(V \rightarrow X, \omega_{V}\right)$ : one has the Grassmann bundle $\operatorname{Gr}_{X}(2, V) \rightarrow X$ and the open subbundle $\mathbb{H P}(V) \rightarrow X$ of 2-planes for which $\omega_{V}$ restricts to a nondegenerate form. Just as for $\mathbb{A P}^{n}$, we have restriction $\tilde{E}_{2 V} \rightarrow \mathbb{A P}(V)$ of the tautological 2-plane bundle, which gives us the Borel class $b_{1}\left(\tilde{E}_{2 V}\right)$, defined as the Euler class $e\left(\tilde{E}_{2 V}\right) \in \mathcal{E}^{4,2}(\mathbb{H P}(V))$. The main result of Panin-Walter is that $\mathcal{E}^{* *}(\mathbb{H P}(V))$ is a free $\mathcal{E}^{* *}(X)$-module with basis the powers $b_{1}\left(\tilde{E}_{2 V}\right), \ldots, b_{1}\left(\tilde{E}_{2 V}\right)^{n}$. They can then apply a suitably modified version of the Grothendieck approach to define the higher Borel classes $b_{i}(V) \in \mathcal{E}^{4 i, 2 i}(X), i=1, \ldots, n$.

This is fine for symplectic bundles, but what about an arbitrary vector bundle $V \rightarrow X$ without a symplectic structure? Here one has an analogy with the Pontryagin classes of real topological vector bundles. The Pontryagin classes $p_{i}(V)$ for a real vector bundle $V \rightarrow B$ are defined by taking the even Chern classes of the complexification of $V$ : $p_{i}(V)=(-1)^{i} c_{2 i}\left(V_{\mathbb{C}}\right) \in H^{4 i}(B, \mathbb{Z})$. The odd Chern classes $c_{2 i+1}\left(V_{\mathbb{C}}\right)$ are all 2-torsion, so the $p_{i}(V)$ have better properties, such as satisfying a Whitney formula, after inverting 2.

In the motivic world, we symplectify a vector bundle $V \rightarrow$ $X$ by forming $V \oplus V^{\vee}$ with the canonical symplectic form $\omega(L, v)=L(v), \omega\left(v, v^{\prime}\right)=0=\omega\left(L, L^{\prime}\right)$ for $v, v^{\prime}$ sections of $V, L, L^{\prime}$ sections of the dual bundle $V^{\vee}$. We then define

$$
p_{i}(V):=(-1)^{i} b_{2 i}\left(\left(V \oplus V^{\vee}, \omega\right)\right) \in \mathcal{E}^{8 i, 4 i}(X) .
$$

Just as the topological Pontryagin classes are better behaved after inverting 2, the motivic Pontryagin classes are better behaved after inverting $\eta$, since the odd Borel classes are $\eta$-torsion. We also note that as the symplectic form for a rank $2 n$ symplectic vector bundle $(V \rightarrow X, \omega)$ defines an isomorphism $\omega^{n}: \operatorname{det} V \rightarrow \mathcal{O}_{X}$, every symplectic vector bundle is an SL-vector bundle, and thus an SL-oriented theory is also Sp-oriented. We therefore have a good theory of Pontryagin classes for arbitrary vector bundles with values in a given SL-oriented theory $\mathcal{E}$ for which $\eta$ is already invertible.

We do have a wealth of theories that do not die after inverting $\eta$, ranging in complexity from the EilenbergMacLane spectrum $\operatorname{EM}\left(\mathcal{K}_{*}^{M W}\right)$ to hermitian $K$-theory $\mathrm{KQ}$, special linear cobordism MSL, or symplectic cobordism MSp. In topology, all the trouble is at the prime 2, and as the real realization of $\eta$ is multiplication by 2 , it seems reasonable to look first at these more complicated theories after inverting $\eta$.

The isomorphism of $\mathcal{K}_{*}^{M W}\left[\eta^{-1}\right]$ with the sheaf of Witt rings $\mathcal{W}$ leads to the identification $\operatorname{EM}\left(\mathcal{K}_{*}^{M W}\right)\left[\eta^{-1}\right] \cong$ $\operatorname{EM}\left(\mathcal{W}_{*}\right)$, where $\mathcal{W}_{*}$ is the homotopy module $\mathcal{W}_{n}=\mathcal{W}$ We also have the simple description of $\operatorname{EM}\left(\mathcal{W}_{*}\right)$-theory and $\mathcal{W}$-cohomology: $\operatorname{EM}\left(\mathcal{W}_{*}\right)^{a, b}(X)=H^{a-b}(X, \mathcal{W})$. As $W(\mathbb{R})=\mathbb{Z}$, one can view $H^{*}(-, \mathcal{W})$ as a good analog of singular cohomology $H^{*}(-, \mathbb{Z}[1 / 2])$ via the real realization. In fact, it follows from Bachmann's result on the real realization (Theorem 2) that in $\operatorname{SH}(\mathbb{R}), \operatorname{EM}\left(\mathcal{K}_{*}^{M W}\right)\left[\rho^{-1}\right]$ represents usual singular cohomology of the real points. Thus, as the real realization sends $\eta$ to $2, H^{*}(X, \mathcal{W}[1 / 2])=$ $H^{*}(X(\mathbb{R}), \mathbb{Z}[1 / 2])$ for $X$ a smooth $\mathbb{R}$-scheme.

Also, the corresponding Pontryagin classes $p_{i}(V)$ for a vector bundle $V \rightarrow X$ live in $\operatorname{EM}\left(\mathcal{W}_{*}\right)^{8 i, 4 i}(X)=$ $H^{4 i}(X, \mathcal{W})$, another good analogy with the topological setting. I suspect that the motivic Pontryagin class $p_{i}(V) \in$ $H^{4 i}(X, \mathcal{W})$ goes over to the topological one $p_{i}(V(\mathbb{R})) \in$ $H^{4 i}(X(\mathbb{R}), \mathbb{Z}[1 / 2])$ under real realization, but as far as $\mathrm{I}$ know this has not been checked. In any case, the $\eta$ invertible theories in $\mathrm{SH}(k)$ are useful tools in pursuing analogies between motivic homotopy theory and the classical setting via real realizations.

The theory of characteristic classes for theories has been extensively studied by Ananyevskiy [2]. Here are a few of his results. The first one says that for SL-vector bundles, the Pontryagin classes and (for even rank bundles) the Euler class generate all characteristic classes in an $\eta$-inverted, SLoriented theory.

Theorem 5 (Ananyevskiy [2, Theorem 10]). Let $\mathcal{E} \in \mathrm{SH}(k)$ be an $\eta$-inverted, SL-oriented commutative ring spectrum. Then

$$
\begin{aligned}
& \mathcal{E}^{* *}\left(\mathrm{BSL}_{n}\right) \\
& = \begin{cases}\mathcal{E}^{* *}(k)\left[p_{1}, \ldots, p_{m}, e\right] /\left(p_{m}-e^{2}\right) & \text { for } n=2 m \text { even, } \\
\mathcal{E}^{* *}(k)\left[p_{1}, \ldots, p_{m}\right] & \text { for } n=2 m+1 \text { odd } .\end{cases}
\end{aligned}
$$

Here $p_{i}=p_{i}\left(E_{n}\right)$, where $E_{n} \rightarrow \mathrm{BSL}_{n}$ is the universal vector bundle, and $e=e\left(E_{n}\right)$ is the Euler class. 
As is well known, the projective bundle formula for Chern classes gives rise to the splitting principle, which says that in order to check a natural identity in Chern classes of vector bundles, it suffices to check the identity for Chern classes of direct sums of line bundles. This principle is not available for SL-bundles, as the only line bundle that is an SL-bundle is the trivial line bundle. The following theorem of Ananyevskiy is the next best thing.

Theorem 6 (Ananyevskiy [2, Theorem 6]). Let $\mathcal{E} \in \mathrm{SH}(k)$ be an $\eta$-inverted, SL-oriented commutative ring spectrum. Then the block diagonal embedding $\left(\mathrm{SL}_{2}\right)^{m} \rightarrow \mathrm{SL}_{2 m}$ induces an injection

$$
\mathcal{E}^{* *}\left(\mathrm{BSL}_{2 m}\right) \rightarrow \mathcal{E}^{* *}\left(\left(\mathrm{BSL}_{2}\right)^{m}\right)
$$

Moreover, $\mathcal{E}^{* *}\left(\left(\mathrm{BSL}_{2}\right)^{m}\right)=\mathcal{E}^{* *}(k)\left[e_{1}, \ldots, e_{m}\right]$, where $e_{i}$ is the pullback of the Euler class $e\left(E_{2}\right)$ via the ith projection $\left(\mathrm{BSL}_{2}\right)^{m} \rightarrow \mathrm{BSL}_{2}$. Finally, the inclusion

$$
\begin{aligned}
\mathcal{E}^{* *} & \left(\mathrm{BSL}_{2 m}\right) \\
& =\mathcal{E}^{* *}(k)\left[p_{1}, \ldots, p_{m}, e\right] /\left(p_{m}-e^{2}\right) \rightarrow \mathcal{E}^{* *}(k)\left[e_{1}, \ldots, e_{m}\right] \\
& =\mathcal{E}^{* *}\left(\left(\mathrm{BSL}_{2}\right)^{m}\right)
\end{aligned}
$$

identifies $\mathcal{E}^{* *}\left(\mathrm{BSL}_{2 m}\right)$ with the invariants of $\mathcal{E}^{* *}(k)\left[e_{1}, \ldots, e_{m}\right]$ under the action of the semidirect product $\{ \pm 1\}^{m-1} \rtimes S_{m}$, which acts by sending $\left(e_{1}, \ldots, e_{m}\right)$ to $\left(\epsilon_{1} e_{\sigma(1)}, \ldots, \epsilon_{m} e_{\sigma(m)}\right)$, where $\sigma \in$ $S_{m}$ is a permutation and $\epsilon_{i} \in\{ \pm 1\}$ with $\prod_{i} \epsilon_{i}=1$. Explicitly,

$$
\begin{aligned}
& p\left(E_{2 m}\right) \\
& :=1+p_{1}\left(E_{2 m}\right)+\cdots+p_{m}\left(E_{2 m}\right)=\prod_{i=1}^{m}\left(1+e_{i}^{2}\right), \quad e=\prod_{i=1}^{m} e_{i} .
\end{aligned}
$$

This reduction to rank 2 bundles still leaves open a basic question. Given an $\mathrm{SL}_{n}$-vector bundle $V \rightarrow X$ and a representation $\rho: \mathrm{SL}_{n} \rightarrow \mathrm{SL}_{N}$ one has the associated $\mathrm{SL}_{N^{-}}$ bundle $V^{\rho} \rightarrow X$. What are the Pontryagin classes and Euler class of $V^{\rho}$ in terms of those of $V$ ? For the Chern classes, this is answered by the classical splitting principle and the theory of symmetric functions. Ananyevskiy's $\mathrm{SL}_{2}$ splitting principle reduces one to understanding the characteristic classes of $\operatorname{Sym}^{n} E_{2}$, with $E_{2} \rightarrow \mathrm{BSL}_{2}$ the tautological bundle.

The solution lies in a further splitting principle: reduction to the normalizer $N$ of the maximal torus $T \subset \mathrm{SL}_{2} . T$ is isomorphic to $\mathbb{G}_{m}$ via

$$
t \mapsto\left(\begin{array}{cc}
t & 0 \\
0 & t^{-1}
\end{array}\right)
$$

and $N$ is generated by $T$ and the element $\sigma$,

$$
\sigma=\left(\begin{array}{cc}
0 & 1 \\
-1 & 0
\end{array}\right)
$$

Based on a motivic version of the classical Becker-Gottlieb transfer, we show in [10] that for any $\mathcal{E} \in \mathrm{SH}(k)$, the pullback via $\pi: B N \rightarrow \mathrm{BSL}_{2}$ induces an injection

$$
\pi^{*}: \mathcal{E}^{* *}\left(\mathrm{BSL}_{2}\right) \rightarrow \mathcal{E}^{* *}(B N) \text {. }
$$

This is useful, as the irreducible representations of $N$ are all one- or two-dimensional, so the bundle $\pi^{*} \operatorname{Sym}^{n} E_{2}$ splits as a direct sum of bundles of rank at most two. One can write these bundles explicitly, and we have computed their Euler classes in Witt cohomology. This yields the following formula, inspired by an analogous formula of OkonekTeleman for real topological bundles.

Theorem 7 (Levine [10, Theorem 8.1]). Suppose $k$ has characteristic 0 or characteristic $p>n$. Then in $H^{n+1}\left(\mathrm{BSL}_{2}, \mathcal{W}\right)$, we have

$$
e\left(\operatorname{Sym}^{n} E_{2}\right)= \begin{cases}0 & \text { for } n \text { even }, \\ n ! ! e\left(E_{2}\right)^{m+1} & \text { for } n=2 m+1 \text { odd } .\end{cases}
$$

Here $n ! !=n(n-2) \cdots 3 \cdot 1$ for $n$ odd. Moreover, the total Pontryagin class $p\left(\operatorname{Sym}^{n} E_{2}\right) \in H^{*}\left(\mathrm{BSL}_{2}, \mathcal{W}\right)$ is given by

$$
p\left(\operatorname{Sym}^{n} E_{2}\right)=\prod_{i=0}^{[n / 2]}\left(1+(n-2 i)^{2} e\left(E_{2}\right)^{2}\right) .
$$

We note in [10, Example 8.2] that the above computation can be used to give a "count" of the lines on a general hypersurface of degree $2 d-1$ in $\mathbb{P}^{d+1}$ as

$$
C_{d}=n ! !\langle 1\rangle+\frac{1}{2}\left(N_{d}-n ! !\right) h \in \mathrm{GW}(k),
$$

where $h$ is the hyperbolic form $\langle 1\rangle+\langle-1\rangle, N_{d}$ is the degree of the top Chern class $c_{2 d}\left(\operatorname{Sym}^{2 d+1} E_{2,2 d+2}^{\vee}\right)$, and $E_{2, d+2} \rightarrow$ $\operatorname{Gr}(2, d+2)$ is the tautological bundle on the Grassmannian of 2-planes in $d+2$-space. The rank of $C_{d}$ gives the classical count of the number of lines on a general hypersurface of degree $2 d-1$ in $\mathbb{P}^{d+1}$, while the signature of $C_{d}$ has an interpretation of a signed count of real lines on a general real hypersurface of degree $2 d-1$ in $\mathbb{P}^{d+1}$, another example of how the $\eta$-invertible theories relate to topology over the reals.

The quadratic form $C_{d}$ itself is constructed as the pushforward of the Euler class

$$
e\left(\operatorname{Sym}^{2 d-1} E_{2, d+2}\right) \in H^{2 d}\left(\operatorname{Gr}(2, d+2), \mathcal{K}_{2 d}^{M W}\right)
$$

to $\mathrm{GW}(k)=H^{0}\left(\operatorname{Spec} k, \mathcal{K}_{0}^{M W}\right)$, that is, the quadratic degree of the Euler class. The case of lines on a cubic was treated earlier using different motivic methods by KassWickelgren [9]. We are hopeful that pursuing these invariants in Witt cohomology and other motivic theories, one can enrich classical enumerative geometry by providing invariants that have interesting arithmetic content through the theory of quadratic forms.

In another direction, the computation of the Euler class of symmetric powers given by Theorem 7 is limited to the case of the classes in Witt cohomology. As $H^{*}(-, \mathcal{W})$ is in some sense an analogy of classical singular cohomology, one would expect a more complicated behavior for the characteristic classes in other theories, such as KQ, MSL, or MSp, even after inverting $\eta$. For oriented theories, the 
degree of complexity is captured by the associated formal group law. Is there such an algebraic invariant of an SLoriented, $\eta$-inverted theory?

Yet another question involves comparing the plus and minus parts of theories. For example, Witt cohomology is formed by inverting $\eta$ on bi-graded Milnor-Witt cohomology $H^{*}\left(-, \mathcal{K}_{*}^{M W}\right)$. This latter theory (in appropriate degrees) specializes to the Chow ring by killing $\eta$ and to Witt cohomology by inverting $\eta$; one has similar behavior for the other theories such as KQ, MSL, and MSp. Can one exploit this to find comparisons between behavior of characteristic classes or solutions of enumerative problems over $\mathbb{C}$ and over $\mathbb{R}$ ? There are for instance conjectures of Itenberg-Kharlamov-Shustin comparing the growth of classical enumerative invariants with their real counterparts [8]. Can these questions be approached using motivic methods?

\section{References}

[1] Ananyevskiy A, Levine M, Panin I. Witt sheaves and the $\eta$-inverted sphere spectrum. J. Topol. 10 (2017), no. 2, 370385. MR3653315

[2] Ananyevskiy A. The special linear version of the projective bundle theorem. Compos. Math. 151 (2015), no. 3, 461501. MR3320569

[3] Bachmann T. Motivic and real étale stable homotopy theory. Compos. Math. 154 (2018), no. 5, 883-917. MR3781990

[4] Cisinski D-C, Déglise F. Triangulated categories of mixed motives, arXiv 0912.2110 [math.AG], 2009.

[5] Hoyois M. From algebraic cobordism to motivic cohomology. J. Reine Angew. Math. 702 (2015), 173-226. MR3341470

[6] Isaksen DC, Xu Z. Motivic stable homotopy and the stable 51 and 52 stems. Topology Appl. 190 (2015), 31-34. MR3349503

[7] Isaksen DC, Østvær PA. Motivic stable homotopy groups. Preprint, 2018, arXiv:1811.05729 [math.AT].

[8] Itenberg IV, Kharlamov VM, Shustin EI. Logarithmic equivalence of the Welschinger and the Gromov-Witten invariants. Uspekhi Mat. Nauk 59 (2004), no. 6(360), 85-110; translation in Russian Math. Surveys 59 (2004), no. 6, 10931116. MR2138469

[9] Kass JL, Wickelgren K. An arithmetic count of the lines on a smooth cubic surface. Preprint, 2017, arXiv:1708.01175 [math.AG], [math.AT]. MR3752384

[10] Levine M. Motivic Euler characteristics and Witt-valued characteristic classes. Nagoya Math. J. Published online by Cambridge University Press, 22 March 2019. https:// doi .org/10.1017/nmj . 2019.6.

[11] Morel F. An introduction to $\mathbb{A}^{1}$-homotopy theory. Contemporary Developments in Algebraic K-theory, 357-441, ICTP Lect. Notes, XV, Abdus Salam Int. Cent. Theoret. Phys., Trieste, 2004. MR2175638

[12] Panin I, Pimenov K, Röndigs O. A universality theorem for Voevodsky's algebraic cobordism spectrum. Homology, Homotopy Appl. 10 (2008), no. 2, 211-226. MR2475610
[13] Panin I, Walter C. On the motivic commutative ring spectrum BO. Algebra $i$ Analiz 30 (2018), no. 6, 43-96. MR3882540

[14] Panin I, Walter C. Quaternionic Grassmannians and Borel classes in algebraic geometry. Preprint, 2010 (rev. 2018), arXiv:1011.0649 [math.AG].

[15] Röndigs O, Spitzweck M, Østvær PA. The first stable homotopy groups of motivic spheres. Ann. of Math. (2) 189 (2019), no. 1, 1-74. MR3898173

[16] Röndigs O, Østvær PA. Motives and modules over motivic cohomology. C. R. Math. Acad. Sci. Paris 342 (2006), no. 10, 751-754. MR2227753

[17] Morel F, Voevodsky V. $\mathbb{A}^{1}$-homotopy theory of schemes. Inst. Hautes Études Sci. Publ. Math. No. 90 (1999), 45-143 (2001). MR1813224

[18] Voevodsky V. $\mathbb{A}^{1}$-homotopy theory. Proceedings of the International Congress of Mathematicians, Vol. I (Berlin, 1998). MR1648048

[19] Wang G, Xu Z. The triviality of the 61-stem in the stable homotopy groups of spheres. Ann. of Math. (2) 186 (2017), no. 2, 501-580. MR3702672

[20] Yakerson $M$. The unit map of the algebraic special linear cobordism spectrum. Preprint, 2019, arXiv:1908.03859 [math.KT].

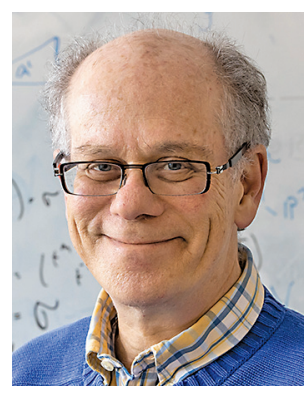

Marc Levine

Credits

Opener image is courtesy of Getty.

Author photo is courtesy of University of Duisberg-Essen. 